\title{
Review Article \\ Self-Eating: Friend or Foe? The Emerging Role of Autophagy in Idiopathic Pulmonary Fibrosis
}

\author{
George A. Margaritopoulos, ${ }^{1}$ Eliza Tsitoura, ${ }^{2}$ Nikos Tzanakis, ${ }^{1}$ Demetrios A. Spandidos, ${ }^{2}$ \\ Nikos M. Siafakas, ${ }^{1}$ George Sourvinos, ${ }^{2}$ and Katerina M. Antoniou ${ }^{1}$ \\ ${ }^{1}$ Interstitial Lung Disease Unit, University Hospital of Heraklion, 71110 Heraklion, Crete, Greece \\ ${ }^{2}$ Laboratory of Virology, Medical School, University of Crete, 71110 Heraklion, Crete, Greece
}

Correspondence should be addressed to George A. Margaritopoulos; gmargaritop@yahoo.gr

Received 27 December 2012; Revised 27 February 2013; Accepted 27 February 2013

Academic Editor: Sharbel Weidner Maluf

Copyright (C) 2013 George A. Margaritopoulos et al. This is an open access article distributed under the Creative Commons Attribution License, which permits unrestricted use, distribution, and reproduction in any medium, provided the original work is properly cited.

\begin{abstract}
Idiopathic pulmonary fibrosis is the most common and severe form of idiopathic interstitial pneumonias. Despite an exponential increase in our understanding of potentially important mediators and mechanisms, the pathogenesis remains elusive, and little therapeutic progress has been made in the last few years. Mortality in 3-5 years is still $50 \%$. Autophagy, a highly conserved homeostatic mechanism necessary for cell survival, has been recently implicated in the pathogenesis of pulmonary disorders. In this paper we aim to highlight some key issues regarding the process of autophagy and its possible association with the pathogenesis of idiopathic pulmonary fibrosis.
\end{abstract}

\section{Introduction}

Idiopathic pulmonary fibrosis (IPF) is a chronic and progressive fibrosing interstitial pneumonia of unknown cause whose pathogenesis, despite recent advances, is still not fully understood [1]. It is currently believed that IPF is the result of repeated injuries in different sites of the alveolar epithelium with dysregulation of cellular homeostasis followed by aberrant wound healing and inadequate repair of the epithelial damage. Median survival from the time of diagnosis is approximately 3 years, and surprisingly the mortality rate exceeds that of many cancers [2]. Lung transplantation is the only therapeutic approach which can affect survival, but it is limited to highly selected patients. However, recent clinical trials have yielded encouraging results regarding the use of pirfenidone and $\mathrm{N}$-acetyl-cysteine in mild to moderate disease $[3,4]$.

In view of the limited current knowledge and poor survival of the disease, the need for an elucidation of the pathogenesis becomes imperative. Recently, the process of autophagy, a term derived from the Greek and meaning "selfeating," has been implicated in the pathogenetic pathway of IPF $[5,6]$. Autophagy is a highly conserved homeostatic mechanism by which cells transport damaged proteins and organelles such as mitochondria to lysosomes for degradation in both health and disease conditions. It contributes to cellular homeostasis by (a) providing an alternative source of metabolic fuel, (b) removing damaged cellular components which are toxic to the cell such as dysfunctional mitochondria or aggregated proteins, and (c) promoting cell death [7].

In previous studies, the term of selective autophagy has been introduced in order to underline the selective targeting of cargoes for degradation. Mitophagy refers to selective digestion of mitochondria, and xenophagy refers to selective degradation of invading pathogens and bacteria $[8,9]$. The selective degradation of protein aggregates is regulated by p62, a cytosolic chaperone protein.

Most of the current knowledge regarding the role of autophagy in cell homeostasis was obtained by studies in yeast and mice, whereas its role in human diseases has only been slightly investigated and appears to be highly pleiotropic as it may either represent an adoptive prosurvival response or, if deregulated, promote cell death and morbidity. Autophagic cell death differs from apoptotic cell-death; the former does 
not involve caspase activation. Nonetheless, the two processes can coexist [10].

In this paper, we aim to highlight the current knowledge regarding the process of autophagy and its role in various pulmonary disorders with a particular focus on IPF.

\section{Classes and Regulation of Autophagy}

There are three mechanisms by which autophagy can occur. Microautophagy involves a nonselective surrounding of cytoplasmic components directly by lysosomal membranes. The chaperone-mediated autophagy involves a selective transport to lysosomes of cargoes that contain a specific pentapeptide motif (KFERQ). Macroautophagy is characterized by the presence of autophagosome, a double-membraned vesicle that surrounds the damaged component to be degraded following fusion with endosomes and lysosomes. Chaperone mediated autophagy and microautophagy are less studied whereas macroautophagy (referred as autophagy hereafter) is the best studied mechanism since autophagosomes are easy to detect with fluorescence and electron microscopy [11]. The process consists of in four distinct steps: (a) the formation of an isolation membrane, (b) the formation of an autophagosome with engulfment of the cargo, (c) the fusion of the autophagosome with a lysosome, and (d) the degradation of the cargo by lysosomal enzymes with regeneration of metabolic precursor molecules to be used for anabolic pathways [7].

A series of autophagy-related genes (Atg) are involved in the regulation of the process. Beclin-1, an interacting protein, in complex with class III phosphatidylinositol 3kinase (PI3 K) and Atg14 acts as a major positive regulator of autophagy [12]. The rapamycin-sensitive mammalian target of rapamycin (mTOR)/class I PI3 K pathway acts as a major negative regulator of autophagy $[13,14]$. Autophagosome formation requires two ubiquitin-like conjucation systems: the Atg5-Atg12 conjucation system and the Atg8 (microtubuleassociated protein-1 light chain [LC] (3) conjugation system by which LC3 is converted from LC3-I (free form) to LC3 II (conjugated to phosphaditylethanolamine form) a step which is considered critical for autophagosome formation [15-17]. The fusion of autophagosome with lysosome requires the involvement of a GTPase termed Rab-7 and lysosomemediated membrane protein (LAMP) -1 and -2 .

Autophagy can be measured with various methods, and each one has its advantages and limitations. Electron microscopy can visualize early-stage autophagosomes but is less sensitive for the visualization of late-stage autophagosomes [18]. Fluorescence-based methods such as the use of green fluorescent protein (GFP)-LC3 are also used. They are based on the fact that when autophagy is induced and LC3b becomes part of the newly formatted autophagosome, the GFP-LC3 changes its cellular localization from a diffuse cytosolic pattern to a punctuate pattern [19]. Western blot analysis has demonstrated that LC3b-II correlates well with the number of autophagosomes and, thus, with autophagic activity [20]. Western blot analysis of p62, a cytosolic chaperone protein that has an LC3b binding domain [21], can also be used as an increase of p62 levels is associated with the reduced autophagic activity [22]. These methods have the disadvantage of evaluating autophagy in a certain time point (i.e., static measures) and may not reflect the autophagic activity properly as an increase of either the number of autophagosomes or the levels of LC3b-II may be due to the enhanced induction of autophagosome formation or to inhibited fusion with lysosomes which in reality means low autophagic flux. In order to distinguish between these two options, autophagic flux assays are used (i.e., dynamic measures). In cell cultures, LC3b-II levels are measured with Western blot in presence and absence of inhibitors of autophagic degradation such as chloroquine, leupeptin, and bafilomycin-A [23]. Another pitfall regarding the measurement of autophagy in lung diseases is that the process is cell-dependent as it was shown in COPD [24, 25] where it is enhanced in the epithelium and impaired in alveolar macrophages. Thus, the study of autophagy in samples such as whole lung homogenates may not represent what happens in a specific subset of cells. A combination of static and dynamic methods is currently recommended with a careful definition of the type of cell in which autophagy is being measured [7].

\section{Autophagy in Pulmonary Diseases}

Autophagy has been implicated in the pathogenesis of several pulmonary diseases (Table 1) and represents a potential therapeutic target in current and future clinical trials (Table 2) [26]. Chronic obstructive pulmonary disease (COPD) is the best studied lung disorder regarding the role of autophagy and represents an example of the cell-specific role of autophagy in the context of the same disease. In lung biopsy samples, it was observed that the number of autophagosomes as well as the levels of LC3b-II and other autophagyrelated proteins was increased and moreover was correlated with disease severity [27]. Similar results were obtained in lung epithelial cell lines and fibroblasts exposed to cigarette smoking extract (CSE) which is the primary causative agent of COPD [27, 28]. Genetic depletion of Beclin-1 and LC3b decreased cell death in exposed cells, and mice deficient in LC3b did not develop emphysema after exposure to CSE $[24,27,29]$. On the other hand, in alveolar macrophages, autophagic flux was impaired, and the effect of smoking was further supported by the fact that similar changes were observed in alveolar macrophages of non smokers exposed to cigarette smoking [25]. Therefore, in conclusion, enhanced autophagy in epithelial cells has a deleterious effect by promoting cell death and developing of emphysema, and impaired autophagy in alveolar macrophages contributes to the deficient transport of bacteria to lysosome, namely, xenophagy, and to the higher propensity of COPD patients to bacterial acute respiratory infections which lead to acute exacerbations.

Cystic fibrosis (CF) is a disease characterized by mutations of the cystic fibrosis transmembrane conductance regulator (CFTR) gene which is associated to intracellular accumulation of misfolded proteins that are supposed to be cleared by autophagy. It has been observed that in CF human epithelial cell, mutant CFTR proteins impair autophagosome formation via depletion of Beclin-1 [30] and increase 
TABLE 1: Autophagy and pulmonary diseases.

\begin{tabular}{|c|c|c|c|}
\hline Study & Model & Disease & Stimulus/effect \\
\hline Chen et al. 2008 [24] & in vivo/in vitro & COPD & CS induces autophagy in epithelial cells. \\
\hline Monick et al. 2010 [25] & in vitro & COPD & $\begin{array}{l}\text { CS-induced cell death and emphysema are regulated by LC3 } \beta \\
\text { activity. }\end{array}$ \\
\hline Chen et al. 2010 [29] & in vivo/in vitro & COPD & CS decreases autophagy in alveolar macrophages. \\
\hline Luciani et al. 2010 [30] & in vivo/in vitro & $\mathrm{CF}$ & Defective autophagy in CF \\
\hline Abdulrahman et al. 2011 [31] & in vivo/in vitro & $\mathrm{CF}$ & $\begin{array}{l}\text { Treatment with rapamycin induces autophagy and reduces the } \\
\text { burden of } B \text {. cenocepacia. }\end{array}$ \\
\hline Singh et al. 2006 [33] & in vitro & MTB infection & $\begin{array}{l}\text { Induction of autophagy by IFN } \gamma \text { or rapamycin enhances } \\
\text { mycobacteria killing. }\end{array}$ \\
\hline Gutierez et al. 2004 [34] & in vitro & MTB infection & $\begin{array}{l}\text { Induction of autophagy by IFN } \gamma \text { eliminates mycobacteria } \\
\text { through an IRGM mechanism. }\end{array}$ \\
\hline Lee et al. 2011 [35] & in vivo/in vitro & $\mathrm{PH}$ & Autophagy protects against hypoxia-induced $\mathrm{PH}$. \\
\hline Parhitko et al. 2011 [36] & in vivo/in vitro & TSC & $\begin{array}{l}\text { Genetic-pharmacologic inhibition of autophagy blocks } \\
\text { autophagy in TSC. }\end{array}$ \\
\hline Gills et al. 2007 [39] & in vivo/in vitro & Lung cancer & $\begin{array}{l}\text { Nelfinavir activates autophagy and exhibits antiproliferative } \\
\text { activity in lung cancer. }\end{array}$ \\
\hline Mi et al. $2011[80]$ & in vivo/in vitro & $\mathrm{PF}$ & $\begin{array}{l}\text { Antagonism of IL-17A induces autophagy and protects against } \\
\text { fibrosis. }\end{array}$ \\
\hline Patel et al. 2012 [5] & in vivo/in vitro & $\mathrm{IPF}$ & $\begin{array}{l}\text { TGF- } \beta 1 \text { inhibits autophagy in lung fibrobasts. Rapamycin } \\
\text { induces autophagy in the BLM model and reduces the degree of } \\
\text { fibrosis. }\end{array}$ \\
\hline Araya et al. 2013 [6] & in vitro & IPF & $\begin{array}{l}\text { Insufficient autophagy promotes myofibroblast differentiation } \\
\text { and collagen deposition. }\end{array}$ \\
\hline
\end{tabular}

COPD: chronic obstructive pulmonary disease, CS: cigarette smoking, CF: cystic fibrosis, MTB: mycobacterium tuberculosis, IFN $\gamma$ : interferon $\gamma$, PH: pulmonary hypertension, TSC: tuberous sclerosis complex, PF: pulmonary fibrosis, IPF: idiopathic pulmonary fibrosis, TGF- $\beta 1$ : transforming growth factor$\beta 1$, BLM: bleomycin.

TABLE 2: Clinical trials targeting autophagy in pulmonary diseases.

\begin{tabular}{|c|c|c|c|c|}
\hline Identifier & Condition & Intervention & Phase & Status \\
\hline NCT00969306 & SCLC & $\begin{array}{l}\text { Chloroquine, } \\
\text { A-CQ100 }\end{array}$ & Phase 1 & Not yet recruiting \\
\hline NCT00933803 & NSCLC & $\begin{array}{l}\text { Paclitaxel } \\
\text { Carboplatin } \\
\text { Hydroxychloroquine } \\
\text { Bevacizumab }\end{array}$ & $\begin{array}{l}\text { Phase } 1 \\
\text { Phase } 2\end{array}$ & $\begin{array}{c}\text { Active } \\
\text { Not recruiting }\end{array}$ \\
\hline NCT01649947 & $\begin{array}{l}\text { NSCLC } \\
\text { Recurrent } \\
\text { NSCLC }\end{array}$ & $\begin{array}{l}\text { Paclitaxel } \\
\text { Carboplatin } \\
\text { Hydroxychloroquine } \\
\text { Bevacizumab }\end{array}$ & Phase 2 & Recruiting \\
\hline NCT00728845 & $\begin{array}{c}\text { Recurrent } \\
\text { Advanced } \\
\text { NSCLC }\end{array}$ & $\begin{array}{l}\text { Bevacizumab } \\
\text { Carboplatin } \\
\text { Hydroxychloroquine } \\
\text { Paclitaxel }\end{array}$ & $\begin{array}{l}\text { Phase } 1 \\
\text { Phase } 2\end{array}$ & Terminated \\
\hline NCT01687179 & LAM & $\begin{array}{l}\text { Sirolimus and } \\
\text { hydroxychloroquine }\end{array}$ & Phase 1 & Recruiting \\
\hline
\end{tabular}

SCLC: small-cell lung cancer, NSCLC: non-small cell lung cancer, LAM: lymphangioleiomyomatosis.

of reactive oxygen species (ROS) and transglutaminase-2 production which contributes to the excessive inflammation seen in CF. Restoring Beclin-1 restores autophagy measured by LCEb-II and p62 levels and GFP-LC3 puncta and leads to increased clearance of protein aggregates and marked reduction of inflammation in a mouse model of CF [30].
Interestingly, treatment with $\mathrm{N}$-acetylcysteine (NAC) also has beneficial effects suggesting a possible implication of antioxidants in the therapy of CF. Infection by Burkholderia cenocepacia is potentially lethal in patients with CF, and it was observed that it is associated with downregulation of several autophagic genes in alveolar macrophages [31]. Treatment 
with rapamycin induced autophagy and reduced lung inflammation.

Alveolar macrophages provide the first line of defense against invading microbes. Infection by Mycobacterium tuberculosis is also associated with inhibition of the formation of autophagosomes which inhibits mycobacterial killing by alveolar macrophages [32]. Stimulation of alveolar macrophages with interferon- $\gamma($ IFN- $\gamma)$ upregulates a GTPase, namely, IRGM-1, and activates autophagy [33]. Induction of autophagy by rapamycin also enhances mycobacterial killing [34].

Autophagy seems to be implicated in the development of pulmonary hypertension (PH). In lung samples of human $\mathrm{PH}$ and lung vasculature, there was an increased expression of LC3b and GFP-LC3 puncta formation, a marker of autophagosome formation in GFP-LC3-transfected endothelial cells. When mice genetically deficient in LC3b were exposed to chronic hypoxia, they demonstrated an evidence of increased pulmonary hypertension compared to wild-type mice suggesting a protective role of autophagy by limiting hypoxia-dependent vascular cell proliferation [35].

Tuberous sclerosis complex (TSC) is an autosomal dominant tumor suppressor gene syndrome caused by germline mutations in the TSC1 or TSC2 genes. Patients with TSC have multisystem manifestations such as neurologic disease, benign tumors in multiple organs, and pulmonary lymphangioleiomyomatosis (LAM). Genetic and pharmacologic autophagy inhibition blocks tumorigenesis in xenograft and spontaneous models of TSC [36] and hence may represent a potential therapeutic target for TSC.

Autophagy has functional implications in the pathogenesis of cancer, but only few studies have been performed specifically in lung cancer, and the role of autophagy in response to chemotherapeutic agents using cultured human lung A549 adenocarcinoma cells $[37,38]$ has been evaluated. Interestingly, Nelfinavir, an HIV protease inhibitor, exerted pleiotropic biochemical and cellular effects that included induction of endoplasmic reticulum (ER) stress, autophagy, and apoptosis in vitro and in vivo and exhibited antiproliferative activity in lung cancer cells [39].

\section{Autophagy and IPF: Indirect Pathogenetic Links}

A growing body of evidence suggests that there may be a pathogenetic link between IPF and autophagy. Oxidative stress, endoplasmic reticulum (ER) stress, and hypoxia, all mechanisms that participate in the pathogenesis of IPF [40-42], are well-known inducers of autophagy [43-45]. On the other hand, viral infections, which have also been hypothesized to favor the development of fibrosis, seem to have an inhibitory effect on autophagy.

ER is an organelle that serves general functions such as facilitation of protein folding and transport of newly synthesized proteins. Oxidative stress, disturbances in calcium regulation, glucose deprivation, and viral infection can cause ER stress which leads to increase of the unfolded proteins [46]. In neuroblastoma cells, ER stress induces autophagy and is associated with cell survival [47]. However, ER-induced autophagy may be cell specific because it was seen that in colon and prostate cancer cell lines activation of autophagy by chemical inducers reduces cell death, whereas in normal human colon cells and in nontransformed embryonic fibroblasts, it contributes to cell-death [48]. In lung epithelial fibroblast cell lines, autophagy induced by chemicals resulted in increased accumulation of LC3-II and activation of unfolded protein response, a compensatory mechanism to ER stress [49] suggesting a possible protective role of autophagy.

It is now accepted that IPF is the result of multiple injuries in the epithelium which leads to early death of type II alveolar epithelial cells (AECs II) and aberrant wound healing. In cases of familial IPF, it was shown that mutations of the surfactant protein-C gene (SFTRC) lead to accumulation of misfolded proteins, induction of ER stress, and apoptosis of AEC II [50-53]. In sporadic IPF and regardless the absence of mutations, an increase of ER stress and apoptotic markers in AEC II was also observed [54]. Recently, it was also observed that ER stress is implicated in the differentiation of fibroblast into myofibroblast which is considered a key event in the pathogenesis of IPF [40].

Recent evidence suggests that oxidative stress, defined as an imbalance of the generation of ROS in excess of the capacity to neutralize them, promotes autophagy. According to a hypothesis [55], mild levels of oxidative stress activate autophagy in order to eliminate the damaged organelles and thus to promote cell survival. On the other hand, acute or persistent oxidative stress, which has been hypothesized in the pathogenesis of IPF, leads to an increase of intracellular ROS, damage of the lysosomal membrane with intracellular release of potent hydrolases, dysregulation of autophagy with perpetuation of the oxidative injury, and initiation of a vicious circle that leads to apoptotic cell death. In transformed and cancer cell lines treatment with hydrogen peroxide $\left(\mathrm{H}_{2} \mathrm{O}_{2}\right)$ induced autophagy and promoted caspase-independent cell death, whereas knockdown of specific autophagic genes with small interfering RNAs (siRNAs) prevented $\mathrm{H}_{2} \mathrm{O}_{2}$-induced autophagic cell death [56]. Starvation, which is a known inducer of autophagy, is associated with an increase of intracellular ROS and leads to autophagosome formation and autophagic degradation in $\mathrm{CHO}$ and HeLa cells [57]. In the former scenario [56], ROS-induced autophagy led to cell death, whereas in the latter [57] it represented a mechanism which is essential for cell survival. In the model of human bronchial epithelial cells treated with cigarette smoke extract, an increase of intracellular ROS was observed, and the activation of autophagy had a deleterious effect promoting the death of epithelial cells [26].

ROS and markers of oxidative stress are evident in IPF, and levels of ROS are negatively correlated with lung function [58-60]. The overproduction of ROS may cause lung injury and promote a tissue microenvironment which favors fibrosis over regeneration. Glutathione, an antioxidant agent, has been found to be decreased in IPF [61]. NAC is capable of stimulating glutathione synthesis, increasing the intra- and extracellular levels, and thereby partially restoring glutathione levels $[62,63]$. NAC has been found to have favourable effects on the lung function of patients with IPF 
and mainly in those with less progressed disease [4, 64]. However, results of a recent trial showed some conflicting results which need further careful investigation [65].

Autophagy is sensitive to oxygen tension, and hypoxia inducible factor 1- $\alpha$ (HIF 1- $\alpha$ ) has been implicated as a regulator of autophagy and of turnover of damaged mitochondria under hypoxic condition. HIF 1- $\alpha$ target gene, namely, Bcl2/adenovirus E1B $19 \mathrm{kDa}$-interacting protein-3 (BNIP3), also regulates hypoxia-induced autophagy $[45,66]$. However, even in the case of hypoxia, the dual role of autophagy has been emerged as in another study, it was observed that prolonged hypoxia induces autophagic cell death through a BNIP3 dependent mechanism [67].

It is now believed that hypoxia can lead to alveolar epithelial cell apoptosis initiating the cascade of fibrogenesis. In fact, it was observed in both animal models of bleomycininduced fibrosis and in lung tissues of IPF patients that HIF $1-\alpha$ is overexpressed and may exert its role in early stages of fibrogenesis as it was localized in areas of active fibrosis and in normal areas of IPF lung but not within the fibroblastic foci which represent areas of established fibrosis [42].

Autophagy, as part of the host defence system, has been targeted by viral proteins through the evolution of mechanisms of virus escape. The alpha-herpesvirus HSV-1 inhibits autophagy (i.e., xenophagy) through the actions of ICP34.5 and US11 proteins. ICP34.5 protein directly binds Beclin1 leading to the inhibition of the autophagosome formation [68]. As part of the intrinsic antiviral response, infected cells block protein synthesis through the PKR-mediated phosphorylation of eIF2a translation initiation factor, a process that also leads to the upregulation of autophagy [69]. The ICP34.5 and US11 proteins inhibit the phosphorylation of eIF2a at temporally distinct phases of HSV-1 infection, thereby releasing the block to protein synthesis and subsequently inhibiting the induction of autophagy [70, 71]. Similar to alpha-herpesviruses, beta-herpesviruses like hCMV are extremely efficient in blocking autophagosome formation through the TRS1 viral protein which directly interacts and inhibits Beclin1 [72]. Gamma-herpesviruses seem to employ a different mechanism for the inhibition of autophagy which relies on the acquisition of cellular homologues of $\mathrm{Bcl}-$ 2 protein including BHRF1 and BALF-1 of EBV, Orf16 of Kaposi's sarcoma-associated herpesvirus (KSHV), and M11 of murine $\gamma$-herpesvirus 68 ( $\gamma$-HV68) [73]. Bcl-2 protein apart from its antiapoptotic role acts as a potent inhibitor of autophagy through Beclin binding. $\mathrm{Bcl}-2$ is regulated through JNK phosphorylation, upon which $\mathrm{Bcl}-2$ is released from Beclin-1 allowing for the activation of autophagy. The viral Bcl-2 analogues expressed by several gamma-herpesviruses lack the JNK phosphorylation domain, thereby escaping JNK regulation and acting as dominant inhibitors of autophagy.

Several studies have suggested a link between IPF and occult viral infections in the lung, including herpesviruses, adenovirus, hepatitis $C$, and Torque teno virus. It has been hypothesized that these viruses may represent injurious agents in the context of the "multiple hits" hypothesis. The Epstein-Barr virus (EBV) has been detected in both familial and sporadic IPF [74], and EBV protein and DNA expression have been found in IPF lung tissues $[75,76]$. EBV replication has been demonstrated in type II alveolar epithelial cells, and EBV latent membrane protein 1 (LMP-1) expression was detected in the alveolar epithelium in IPF patients, findings that were associated with poor prognosis [77]. Moreover, from the clinical point of view, antiviral treatment has been reported to stabilise the course of IPF [74]. Recently, our group has detected the presence of HSV-1 in patients with fibrotic idiopathic interstitial pneumonias, since the virus presented similar incidence in two different biological samples, tissue, and bronchoalveolar lavage fluid. We have also found that the presence of HSV-1 can enhance fibrosis by inducing the transcription of molecular pathways which promote fibrotic, angiogenetic, wound healing, and innate immunity processes, suggesting a probable role of infectious factors in the pathogenesis of lung fibrosis [78]. Proof of concept experiments of the involvement of herpesviruses in lung fibrosis come from experimental models of pulmonary fibrosis with the MHV-68 murine gamma-herpesvirus. Importantly, experimentally established pulmonary latent infection of mice with MHV-68 could confer higher susceptibility to bleomycin or FITC-induced fibrosis [79] in comparison to the uninfected control mice, thereby supporting a multiple/recurrent hit hypothesis where the herpesvirus presence alters the lung microenvironment and acts as a cofactor in experimentally induced models of pulmonary fibrosis.

\section{Autophagy in IPF}

Recently, Mi et al. have shown that IL17A, a cytokine that induces production of collagen and promotes epithelialmesenchymal transition (EMT) through a transforminggrowth-factor- $\beta 1$ - (TGF- $\beta 1$-) dependent mechanism, inhibits autophagy in mouse epithelial cells [80]. Moreover, they observed that in the murine model of BLM-induced fibrosis, antagonism of IL17A activated autophagy, decreased the production of collagen, attenuated fibrosis and increased survival. This protective effect was abolished after blocking autophagy with 3-methyladenine (3-MA).

Patel et al. studied markers of autophagic activity and concluded that autophagy is not induced in human IPF lungs [5]. More in detail, they observed that the levels of LC3-II were lower and the levels of p62 were higher in IPF lungs compared to controls. Moreover, they observed a decrease in the number of autophagosomes with electron microscope in IPF lungs. In order to provide a plausible answer, they used fibroblast cell lines and showed that TGF- $\beta 1$, a profibrotic cytokine which is overexpressed in IPF, inhibits autophagy. Silencing of LC3 and beclin-1 genes and, hence, inhibiting autophagy enhanced the expression of fibronectin and $\alpha$ smooth cell actin, a marker of myofibroblast which is a key cell in the process of fibrogenesis. In the BLM model, they observed that treatment with rapamycin enhanced autophagy and protected from fibrosis. Based on these observations, the authors concluded that autophagy protects against the development of fibrosis.

A growing body of evidence at both clinical and biological level suggests that IPF is a disease of aging characterised by premature age-related changes in alveolar epithelial cells [81]. It is also accepted that autophagy functions less as tissue 
ages due to insufficient formation of autophagosomes or to deficient elimination after fusion with lysosomes [82]. Recently, Araya et al. also attempted to clarify the role of autophagy in IPF [6]. They suggested that insufficient autophagy leads to epithelial cell senescence as they observed an increased expression of p62 and ubiquitinated proteins, both markers of decreased autophagy as well as an increased expression of p21 which is a marker of cellular senescence. On the other hand and in agreement with previous observations of cell-specific effect of autophagy, they showed that insufficient autophagy in lung fibroblasts leads to the differentiation to myofibroblasts without any effect on their senescence and to increased production of extracellular matrix which are critical steps in the fibrogenetic process.

\section{Conclusion}

Over the last decade, there has been an explosion in the research field regarding the possible mechanisms involved in the pathogenesis of IPF and clinical trials in order to find a therapeutic agent able, at least, to stabilise the course of the disease. Despite all these efforts, pathogenesis of IPF is still not fully understood. A universally accepted therapeutic agent has not yet been found. Recently, it has been suggested that there should be an "oncologic" approach in the pathogenesis of IPF. According to this hypothesis, multiple pathways may be involved simultaneously in the pathogenesis of IPF, and future therapeutic approaches should target these pathways simultaneously [83]. Moreover, this hypothesis has been strengthened by the observation that there are certain similarities between IPF and lung cancer biology [2]. Autophagy has been recently implicated in the pathogenesis of lung diseases, and only few studies exist in the field of IPF, and thus its role is rather obscure. Therefore, more studies are needed in order to clarify the role of autophagy in IPF and in order to develop novel therapeutic approaches.

\section{References}

[1] G. A. Margaritopoulos, M. Romagnoli, V. Poletti, N. M. Siafakas, A. U. Wells, and K. M. Antoniou, "Recent advances in the pathogenesis and clinical evaluation of pulmonary fibrosis," European Respiratory Review, vol. 21, no. 123, pp. 48-56, 2012.

[2] C. Vancheri, "Idiopathic pulmonary fibrosis: an altered fibroblast proliferation linked to cancer biology," Proceedings of the American Thoracic Society, vol. 9, pp. 153-157, 2012.

[3] H. Taniguchi, M. Ebina, Y. Kondoh et al., "Pirfenidone in idiopathic pulmonary fibrosis," European Respiratory Journal, vol. 35, pp. 821-829, 2010.

[4] M. Demedts, J. Behr, R. Buhl et al., "High-dose acetylcysteine in idiopathic pulmonary fibrosis," The New England Journal of Medicine, vol. 353, no. 21, pp. 2229-2242, 2005.

[5] A. S. Patel, L. Lin, A. Geyer et al., "Autophagy in idiopathic pulmonary fibrosis," PLoS One, vol. 7, Article ID e41394, 2012.

[6] J. Araya, J. Kojima, N. Takasaka et al., "Insufficient autophagy in idiopathic pulmonary fibrosis," American Journal of Physiology, vol. 4, no. 1, pp. 56-69, 2013.

[7] J. A. Haspel and A. M. Choi, "Autophagy:a core cellular process with emerging links to pulmonary disease," American Journal of
Respiratory and Critical Care Medicine, vol. 184, pp. 1237-1246, 2011.

[8] I. Kim, S. Rodriguez-Enriquez, and J. J. Lemasters, "Selective degradation of mitochondria by mitophagy," Archives of Biochemistry and Biophysics, vol. 462, no. 2, pp. 245-253, 2007.

[9] B. Levine, "Eating oneself and uninvited guests: autophagyrelated pathways in cellular defense," Cell, vol. 120, no. 2, pp. 159-162, 2005.

[10] B. Levine, S. Sinha, and G. Kroemer, "Bcl-2 family members: dual regulators of apoptosis and autophagy," Autophagy, vol. 4, no. 5, pp. 600-606, 2008.

[11] N. Mizushima and M. Komatsu, "Autophagy: renovation of cells and tissues," Cell, vol. 147, pp. 728-741, 2011.

[12] E. Itakura, C. Kishi, K. Inoue, and N. Mizushima, "Beclin 1 forms two distinct phosphatidylinositol 3-kinase complexes with mammalian Atg14 and UVRAG," Molecular Biology of the Cell, vol. 19, no. 12, pp. 5360-5372, 2008.

[13] M. G. Gutierrez, D. B. Munafó, W. Berón, and M. I. Colombo, "Rab7 is required for the normal progression of the autophagic pathway in mammalian cells," Journal of Cell Science, vol. 117, no. 13, pp. 2687-2697, 2004.

[14] S. Jäger, C. Bucci, I. Tanida et al., "Role for Rab7 in maturation of late autophagic vacuoles," Journal of Cell Science, vol. 117, no. 20, pp. 4837-4848, 2004.

[15] Y. Ohsumi and N. Mizushima, "Two ubiquitin-like conjugation systems essential for autophagy," Seminars in Cell and Developmental Biology, vol. 15, no. 2, pp. 231-236, 2004.

[16] H. He, Y. Dang, F. Dai et al., "Post-translational modifications of three members of the human MAP1LC3 family and detection of a novel type of modification for MAP1LC3B," The Journal of Biological Chemistry, vol. 278, no. 31, pp. 29278-29287, 2003.

[17] Y. Kabeya, N. Mizushima, T. Ueno et al., "LC3, a mammalian homologue of yeast Apg8p, is localized in autophagosome membranes after processing," EMBO Journal, vol. 19, no. 21, pp. 5720-5728, 2000.

[18] J. M. Swanlund, K. C. Kregel, and T. D. Oberley, "Investigating autophagy: quantitative morphometric analysis using electron microscopy," Autophagy, vol. 6, no. 2, pp. 270-277, 2010.

[19] D. J. Klionsky, H. Abeliovich, P. Agostinis et al., "Guidelines for the use and interpretation of assays for monitoring autophagy in higher eukaryotes," Autophagy, vol. 4, no. 2, pp. 151-175, 2008.

[20] N. Mizushima and T. Yoshimori, "How to interpret LC3 immunoblotting," Autophagy, vol. 3, no. 6, pp. 542-545, 2007.

[21] S. Pankiv, T. H. Clausen, T. Lamark et al., "P62/SQSTM1 binds directly to Atg8/LC3 to facilitate degradation of ubiquitinated protein aggregates by autophagy," The Journal of Biological Chemistry, vol. 282, no. 33, pp. 24131-24145, 2007.

[22] G. Bjørkøy, T. Lamark, S. Pankiv, A. Øvervatn, A. Brech, and T. Johansen, "Monitoring autophagic degradation of p62/sqstml," Methods in Enzymology, vol. 451, pp. 181-197, 2009.

[23] N. Mizushima, T. Yoshimori, and B. Levine, "Methods in mammalian autophagy research," Cell, vol. 140, no. 3, pp. 313326, 2010.

[24] Z. H. Chen, H. P. Kim, F. C. Sciurba et al., "Egr-1 regulates autophagy in cigarette smoke-induced chronic obstructive pulmonary disease," PLoS One, vol. 3, no. 10, Article ID e3316, 2008.

[25] M. M. Monick, L. S. Powers, K. Walters et al., "Identification of an autophagy defect in smokers' alveolar macrophages," Journal of Immunology, vol. 185, no. 9, pp. 5425-5435, 2010.

[26] http://www.clinicaltrials.gov/. 
[27] P. K. Hong, X. Wang, Z. H. Chen et al., "Autophagic proteins regulate cigarette smoke-induced apoptosis: protective role of heme oxygenase-1," Autophagy, vol. 4, no. 7, pp. 887-895, 2008.

[28] J. W. Hwang, S. Chung, I. K. Sundar et al., "Cigarette smokeinduced autophagy is regulated by SIRT1-PARP-1-dependent mechanism: implication in pathogenesis of COPD," Archives of Biochemistry and Biophysics, vol. 500, no. 2, pp. 203-209, 2010.

[29] Z. H. Chen, H. C. Lam, Y. Jin et al., "Autophagy protein microtubule associated protein 1 light chain-3b (LC3B) activates extrinsic apoptosis during cigarette smoke-induced emphysema," Proceedings of the National Academy of Sciences of the United States of America, vol. 107, pp. 18880-18885, 2010.

[30] A. Luciani, V. R. Villella, S. Esposito et al., "Defective CFTR induces aggresome formation and lung inflammation in cystic fibrosis through ROS-mediated autophagy inhibition," Nature Cell Biology, vol. 12, no. 9, pp. 863-875, 2010.

[31] B. A. Abdulrahman, A. A. Khweek, A. Akhter et al., "Autophagy stimulation by rapamycin suppresses lung inflammation and infection by burkholderia cenocepacia in a model of cystic fibrosis," Autophagy, vol. 7, pp. 1359-1370, 2011.

[32] V. Deretic, S. Singh, S. Master et al., "Mycobacterium tuberculosis inhibition of phagolysosome biogenesis and autophagy as a host defence mechanism," Cellular Microbiology, vol. 8, no. 5, pp. 719-727, 2006.

[33] S. B. Singh, A. S. Davis, G. A. Taylor, and V. Deretic, "Human IRGM induces autophagy to eliminate intracellular mycobacteria," Science, vol. 313, no. 5792, pp. 1438-1441, 2006.

[34] M. G. Gutierrez, S. S. Master, S. B. Singh, G. A. Taylor, M. I. Colombo, and V. Deretic, "Autophagy is a defense mechanism inhibiting BCG and Mycobacterium tuberculosis survival in infected macrophages," Cell, vol. 119, no. 6, pp. 753-766, 2004.

[35] S. J. Lee, A. Smith, L. Guo et al., "Autophagic protein LC3B confers resistance against hypoxia-induced pulmonary hypertension," American Journal of Respiratory and Critical Care Medicine, vol. 183, no. 5, pp. 649-658, 2011.

[36] A. Parkhitko, F. Myachina, T. A. Morrison et al., "Tumorigenesis in tuberous sclerosis complex is autophagy and p62/sequestosome 1 (SQSTM1)-dependent," Proceedings of the National Academy of Sciences of the United States of America, vol. 108, no. 30, pp. 12455-12460, 2011.

[37] J. H. Zhang, C. D. Fan, B. X. Zhao et al., "Synthesis and preliminary biological evaluation of novel pyrazolo[1,5-a]pyrazin$4(5 \mathrm{H})$-one derivatives as potential agents against A549 lung cancer cells," Bioorganic and Medicinal Chemistry, vol. 16, no. 24, pp. 10165-10171, 2008.

[38] Q. He, B. Huang, J. Zhao, Y. Zhang, S. Zhang, and J. Miao, "Knockdown of integrin $\beta 4$-induced autophagic cell death associated with P53 in A549 lung adenocarcinoma cells," FEBS Journal, vol. 275, no. 22, pp. 5725-5732, 2008.

[39] J. J. Gills, J. LoPiccolo, J. Tsurutani et al., "Nelfinavir, a lead HIV protease inhibitor, is a broad-spectrum, anticancer agent that induces endoplasmic reticulum stress, autophagy, and apoptosis in vitro and invivo," Clinical Cancer Research, vol. 13, no. 17, pp. 5183-5194, 2007.

[40] H. Tanjore, D. S. Cheng, A. L. Degryse et al., "Alveolar epithelial cells undergo epithelial-to-mesenchymal transition in response to endoplasmic reticulum stress," The Journal of Biological Chemistry, vol. 286, pp. 30972-30980, 2011.

[41] C. R. Kliment and T. D. Oury, "Oxidative stress, extracellular matrix targets, and idiopathic pulmonary fibrosis," Free Radical Biology and Medicine, vol. 49, no. 5, pp. 707-717, 2010.
[42] A. Tzouvelekis, V. Harokopos, T. Paparountas et al., "Comparative expression profiling in pulmonary fibrosis suggests a role of hypoxia-inducible factor- $1 \alpha$ in disease pathogenesis," American Journal of Respiratory and Critical Care Medicine, vol. 176, no. 11, pp. 1108-1119, 2007.

[43] T. Yorimitsu, U. Nair, Z. Yang, and D. J. Klionsky, "Endoplasmic reticulum stress triggers autophagy," The Journal of Biological Chemistry, vol. 281, no. 40, pp. 30299-30304, 2006.

[44] R. Kiffin, U. Bandyopadhyay, and A. M. Cuervo, "Oxidative stress and autophagy," Antioxidants and Redox Signaling, vol. 8, no. 1-2, pp. 152-162, 2006.

[45] H. Zhang, M. Bosch-Marce, L. A. Shimoda et al., "Mitochondrial autophagy is an HIF-1-dependent adaptive metabolic response to hypoxia," The Journal of Biological Chemistry, vol. 283, pp. 10892-10903, 2008.

[46] C. Xu, B. Bailly-Maitre, and J. C. Reed, "Endoplasmic reticulum stress: cell life and death decisions," The Journal of Clinical Investigation, vol. 115, no. 10, pp. 2656-2664, 2005.

[47] M. Ogata, S. Hino, A. Saito et al., "Autophagy is activated for cell survival after endoplasmic reticulum stress," Molecular and Cellular Biology, vol. 26, pp. 9220-9231, 2006.

[48] W. X. Ding, H. M. Ni, W. Gao et al., "Differential effects of endoplasmic reticulum stress-induced autophagy on cell survival," The Journal of Biological Chemistry, vol. 282, pp. 47024710, 2007.

[49] S. H. Oh and S. C. Lim, "Endoplasmic reticulum stressmediated autophagy/apoptosis induced by capsaicin (8-methyl$\mathrm{N}$-vanillyl-6-nonenamide) and dihydrocapsaicin is regulated by the extent of c-jun NH2-terminal kinase/extracellular signalregulated kinase activation in WI38 lung epithelial fibroblast cells," Journal of Pharmacology and Experimental Therapeutics, vol. 329, no. 1, pp. 112-122, 2009.

[50] L. M. Nogee, A. E. Dunbar III, S. E. Wert, F. Askin, A. Hamvas, and J. A. Whitsett, "A mutation in the surfactant protein C gene associated with familial interstitial lung disease," The New England Journal of Medicine, vol. 344, no. 8, pp. 573-579, 2001.

[51] A. Q. Thomas, K. Lane, J. Phillips III et al., "Heterozygosity for a surfactant protein $\mathrm{C}$ gene mutation associated with usual interstitial pneumonitis and cellular nonspecific interstitial pneumonitis in one kindred," American Journal of Respiratory and Critical Care Medicine, vol. 165, no. 9, pp. 1322-1328, 2002.

[52] W. J. Wang, S. Mulugeta, S. J. Russo, and M. F. Beers, "Deletion of exon 4 from human surfactant protein $\mathrm{C}$ results in aggresome formation and generation of a dominant negative," Journal of Cell Science, vol. 116, no. 4, pp. 683-692, 2003.

[53] S. Mulugeta, V. Nguyen, S. J. Russo, M. Muniswamy, and M. F. Beers, "A surfactant protein C precursor protein BRICHOS domain mutation causes endoplasmic reticulum stress, proteasome dysfunction, and caspase 3 activation," American Journal of Respiratory Cell and Molecular Biology, vol. 32, no. 6, pp. 521530, 2005.

[54] M. Korfei, C. Ruppert, P. Mahavadi et al., "Epithelial endoplasmic reticulum stress and apoptosis in sporadic idiopathic pulmonary fibrosis," American Journal of Respiratory and Critical Care Medicine, vol. 178, pp. 838-846, 2008.

[55] U. T. Brunk, H. Dalen, K. Roberg, and H. B. Hellquist, "Photooxidative disruption of lysosomal membranes causes apoptosis of cultured human fibroblasts," Free Radical Biology and Medicine, vol. 23, no. 4, pp. 616-626, 1997. 
[56] Y. Chen, E. McMillan-Ward, J. Kong, S. J. Israels, and S. B. Gibson, "Oxidative stress induces autophagic cell death independent of apoptosis in transformed and cancer cells," Cell Death and Differentiation, vol. 15, no. 1, pp. 171-182, 2008.

[57] R. Scherz-Shouval, E. Shvets, E. Fass, H. Shorer, L. Gil, and Z. Elazar, "Reactive oxygen species are essential for autophagy and specifically regulate the activity of Atg4," EMBO Journal, vol. 26, no. 7, pp. 1749-1760, 2007.

[58] I. Rahman, E. Skwarska, M. Henry et al., "Systemic and pulmonary oxidative stress in idiopathic pulmonary fibrosis," Free Radical Biology and Medicine, vol. 27, no. 1-2, pp. 60-68, 1999.

[59] S. Teramoto, Y. Fukuchi, Y. Uejima, C. Y. Shu, and H. Orimo, "Superoxide anion formation and glutathione metabolism of blood in patients with idiopathic pulmonary fibrosis," Biochemical and Molecular Medicine, vol. 55, no. 1, pp. 66-70, 1995.

[60] Z. D. Daniil, E. Papageorgiou, A. Koutsokera et al., "Serum levels of oxidative stress as a marker of disease severity in idiopathic pulmonary fibrosis," Pulmonary Pharmacology and Therapeutics, vol. 21, no. 1, pp. 26-31, 2008.

[61] A. M. Cantin, R. C. Hubbard, and R. G. Crystal, "Glutathione deficiency in the epithelial lining fluid of the lower respiratory tract in idiopathic pulmonary fibrosis," American Review of Respiratory Disease, vol. 139, no. 2, pp. 370-372, 1989.

[62] J. Behr, K. Maier, B. Degenkolb, F. Krombach, and C. Vogelmeier, "Antioxidative and clinical effects of high-dose N-acetylcysteine in fibrosing alveolitis. Adjunctive therapy to maintenance immunosuppression," American Journal of Respiratory and Critical Care Medicine, vol. 156, no. 6, pp. 1897-1901, 1997.

[63] J. Behr, B. Degenkolb, F. Krombach, and C. Vogelmeier, "Intracellular glutathione and bronchoalveolar cells in fibrosing alveolitis: effects of N-acetylcysteine," European Respiratory Journal, vol. 19, no. 5, pp. 906-911, 2002.

[64] J. Behr, M. Demedts, R. Buhl et al., "Lung function in idiopathic pulmonary fibrosis-extended analyses of the IFIGENIA trial," Respiratory Research, vol. 10, article 101, 2009.

[65] Idiopathic Pulmonary Fibrosis Clinical Research Network, G. Raghu, K. J. Anstrom, T. E. King Jr., J. A. Lasky, and F. J. Martinez, "Prednisone, azathioprine, and N-acetylcysteine for pulmonary fibrosis," The New England Journal of Medicine, vol. 366, pp. 1968-1977, 2012.

[66] G. Bellot, R. Garcia-Medina, P. Gounon et al., "Hypoxiainduced autophagy is mediated through hypoxia-inducible factor induction of BNIP3 and BNIP3L via their BH3 domains," Molecular and Cellular Biology, vol. 29, no. 10, pp. 2570-2581, 2009.

[67] M. B. Azad, Y. Chen, E. S. Henson et al., "Hypoxia induces autophagic cell death in apoptosis-competent cells through a mechanism involving BNIP3," Autophagy, vol. 4, no. 2, pp. 195-204, 2008.

[68] A. Orvedahl, D. Alexander, Z. Tallóczy et al., "HSV-1 ICP34.5 confers neurovirulence by targeting the Beclin 1 autophagy protein," Cell Host and Microbe, vol. 1, no. 1, pp. 23-35, 2007.

[69] Z. Tallóczy, W. Jiang, H. W. Virgin 4th et al., "Regulation of starvation- and virus-induced autophagy by the eIF2alpha kinase signaling pathway," Proceedings of the National Academy of Sciences of the United States of America, vol. 99, pp. 190-195, 2002.

[70] B. He, M. Gross, and B. Roizman, "The $\gamma 134.5$ protein of herpes simplex virus 1 complexes with protein phosphatase $1 \alpha$ to dephosphorylate the $\alpha$ subunit of the eukaryotic translation initiation factor 2 and preclude the shutoff of protein synthesis by double-stranded RNA-activated protein kinase," Proceedings of the National Academy of Sciences of the United States of America, vol. 94, no. 3, pp. 843-848, 1997.

[71] M. Mulvey, J. Poppers, D. Sternberg, and I. Mohr, "Regulation of eIF $2 \alpha$ phosphorylation by different functions that act during discrete phases in the herpes simplex virus type 1 life cycle," Journal of Virology, vol. 77, no. 20, pp. 10917-10928, 2003.

[72] M. Chaumorcel, M. Lussignol, L. Mouna et al., "The human cytomegalovirus protein TRS1 inhibits autophagy via its interaction with Beclin 1," Journal of Virology, vol. 86, pp. 2571-2584, 2012.

[73] A. Cuconati and E. White, "Viral homologs of BCL-2: role of apoptosis in the regulation of virus infection," Genes and Development, vol. 16, no. 19, pp. 2465-2478, 2002.

[74] Y. W. Tang, J. E. Johnson, P. J. Browning et al., "Herpesvirus DNA is consistently detected in lungs of patients with idiopathic pulmonary fibrosis," Journal of Clinical Microbiology, vol. 41, no. 6, pp. 2633-2640, 2003.

[75] J. J. Egan, J. P. Stewart, P. S. Hasleton, J. R. Arrand, K. B. Carroll, and A. A. Woodcock, "Epstein-Barr virus replication within pulmonary epithelial cells in cryptogenic fibrosing alveolitis," Thorax, vol. 50, no. 12, pp. 1234-1239, 1995.

[76] J. P. Stewart, J. J. Egan, A. J. Ross et al., "The detection of Epstein-Barr virus DNA in lung tissue from patients with idiopathic pulmonary fibrosis," American Journal of Respiratory and Critical Care Medicine, vol. 159, no. 4, pp. 1336-1341, 1999.

[77] K. Tsukamoto, H. Hayakawa, A. Sato, K. Chida, H. Nakamura, and K. Miura, "Involvement of Epstein-Barr virus latent membrane protein 1 in disease progression in patients with idiopathic pulmonary fibrosis," Thorax, vol. 55, no. 11, pp. 958961, 2000.

[78] I. Lasithiotaki, K. M. Antoniou, V. M. Vlahava et al., "Detection of herpes simplex virus type-1 in patients with fibrotic lung diseases," PLoS One, vol. 6, Article ID e27800, 2011.

[79] K. M. Vannella, T. R. Luckhardt, C. A. Wilke, L. F. van Dyk, G. B. Toews, and B. B. Moore, "Latent herpesvirus infection augments experimental pulmonary fibrosis," American Journal of Respiratory and Critical Care Medicine, vol. 181, no. 5, pp. 465477, 2010.

[80] S. Mi, Z. Li, H. Z. Yang et al., "Blocking IL-17A promotes the resolution of pulmonary inflammation and fibrosis via TGFbetal- dependent and -independent mechanisms," The Journal of Immunology, vol. 187, pp. 3003-3014, 2011.

[81] H. R. Collard, "The age of idiopathic pulmonary fibrosis," American Journal of Respiratory and Critical Care Medicine, vol. 181, no. 8, pp. 771-772, 2010.

[82] A. Terman, "The effect of age on formation and elimination of autophagic vacuoles in mouse hepatocytes," Gerontology, vol. 41, supplement 2, pp. 319-326, 1995.

[83] T. M. Maher, A. U. Wells, and G. J. Laurent, "Idiopathic pulmonary fibrosis: multiple causes and multiple mechanisms?" European Respiratory Journal, vol. 30, no. 5, pp. 835-839, 2007. 

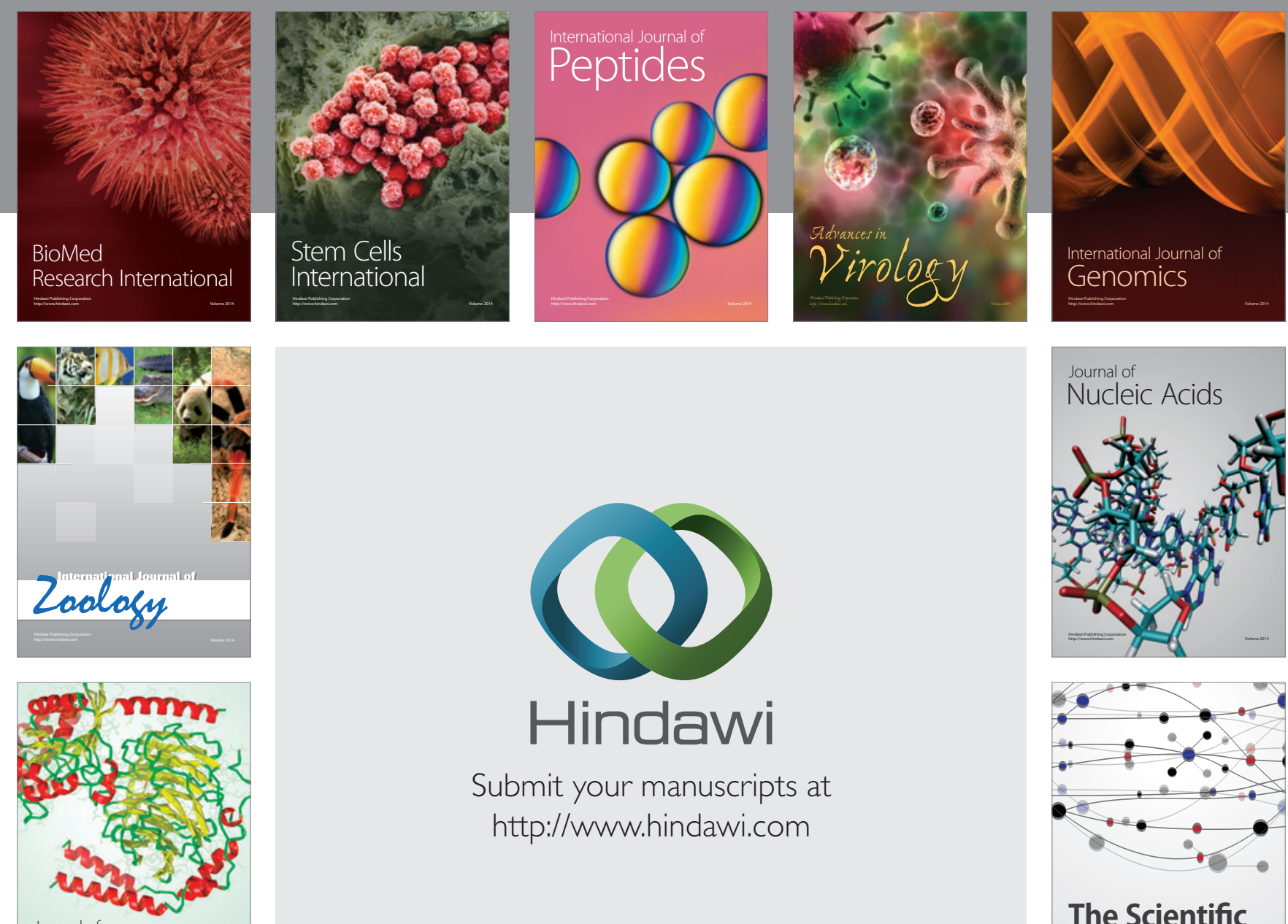

Submit your manuscripts at

http://www.hindawi.com

Journal of
Signal Transduction
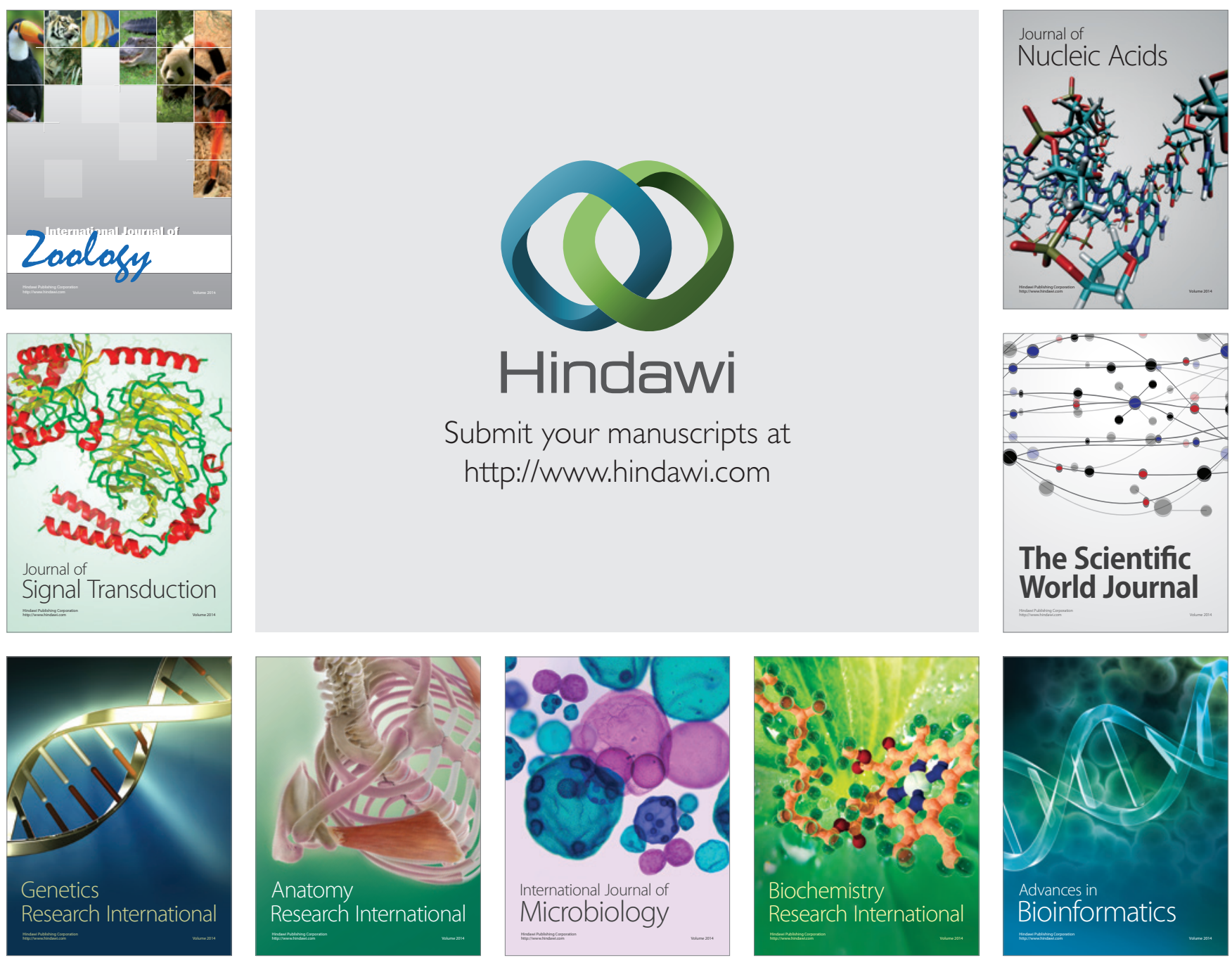

The Scientific World Journal
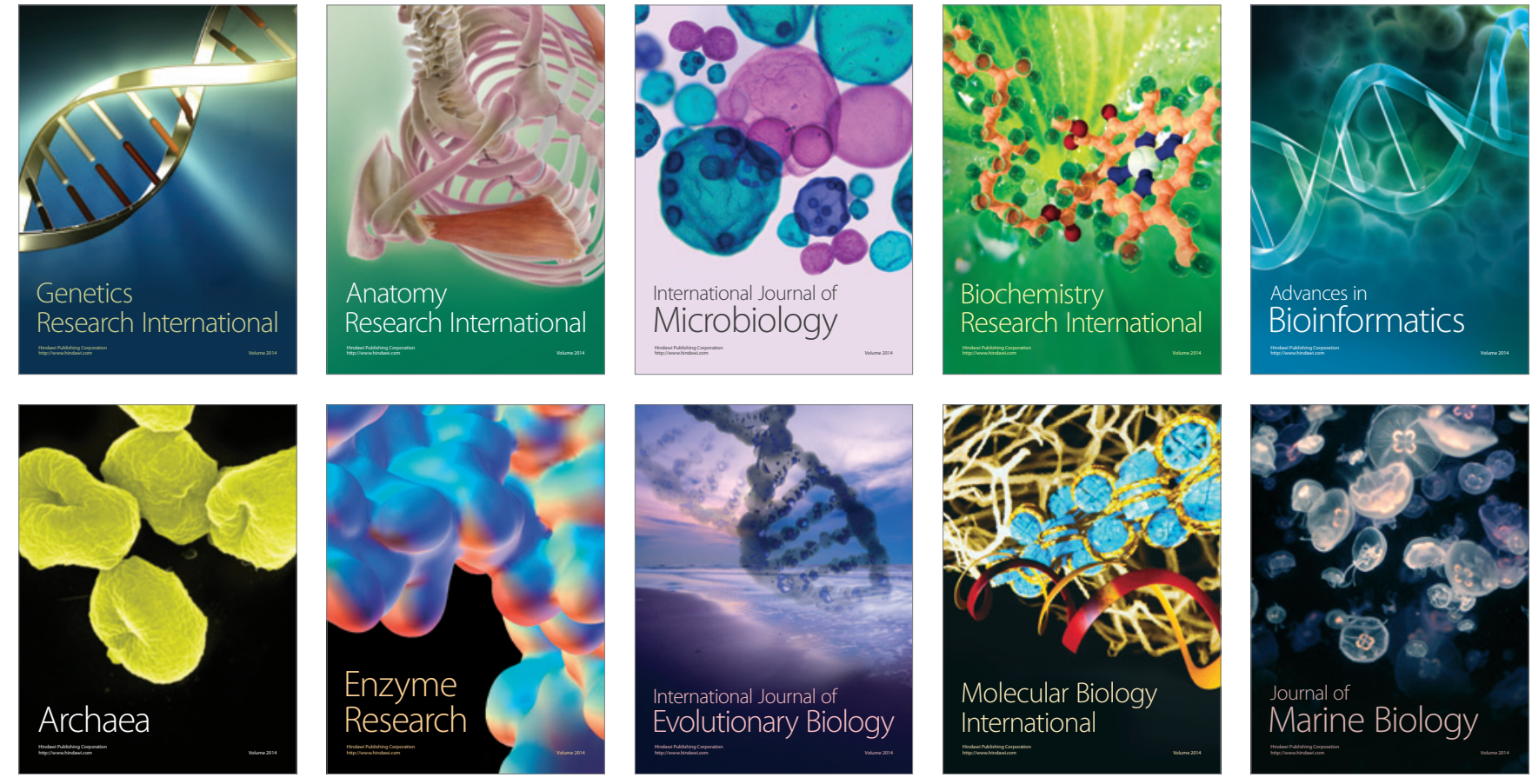